\title{
Light Scattering from Crystalline Superstructure in Tubular-Extruded Poly(1-butene) Films. \\ II. Analysis of Interparticle Interference Effect Based upon a Paracrystal Model
}

\author{
Takeji Hashimoto, Akira Todo*, and Hiromichi KaWAI \\ Department of Polymer Chemistry, Faculty of Engineering, \\ Kyoto University, Kyoto 606, Japan.
}

(Received January 6, 1978)

\begin{abstract}
Light scattering from poly(1-butene) films prepared by tubular extrusion was studied in order to investigate its crystalline superstructure developed by the crystallization from oriented melts. Analysis of the light-scattering patterns together with electron micrographs indicates the existence of a row-nucleated crystalline superstructure whose rows are preferentially oriented at angle $\pm \alpha_{0}$ with respect to the extrusion (ED). The lamellar overgrowth from the nuclei gives a sheaflike crystalline superstructures aligned regularly along the nuclei and nearly side by side with their axes preferentially oriented perpendicular to the ED. The depolarized and polarized components of scattered light from such assembly of the sheaves were analyzed in terms of a paracrystal model of Hosemann type. The analyses have yielded information on (i) average size of the sheaf, (ii) average intersheaf distance and its paracrystalline disorder, and (iii) average orientation of the row nuclei.
\end{abstract}

KEY WORDS Light Scattering / Poly(1-butene) / Tubular-Extruded Films / Crystalline Superstructure / Row-Nucleated Morphology / Interparticle Interference / Paracrystal Model / Oriented Crystallization /

In a previous article, ${ }^{1}$ we studied the morphology and deformation mechanism of the crystalline superstructure of poly(1-butene) films prepared by tubular extrusion. The observed superstructure is typical of the so-called row-nucleated structure developed by crystallization from oriented polymer melts. Figure 1 is a typical electron micrograph of surface replicas of the specimens. The lamellae are supposed to overgrow from "rows" of nucleating points, initially, in a direction normal to the extrusion direction (ED). In the course of its overgrowth, however, the growing front occasionally branches at some distances and angles in space into new lamellae, leading to the development of a diverging sheaf of fibrils as shown in Figure 1.

The sheaves, each of a radius of 3 microns

* On leave from the Research Center, Mitsui Petrochemical Industries, Ltd., Waki-cho, Kuga-gun, Yamaguchi 740, Japan.
$\left(R_{0}=3 \mu\right)$ and half an aperture angle of 30 degrees $\left(\gamma_{0}=30^{\circ}\right)$ are aligned nearly side by side with their axes preferentially oriented perpendicular to the ED. Figure 2 represents the typical $H_{\mathrm{V}}, V_{\mathrm{V}}$, and $H_{\mathrm{H}}$ light-scattering patterns from the specimens and Figure 3, their schematic representations. Important features of the butterfly type $H_{\mathrm{V}}$ scattering pattern and the complex $V_{\mathrm{V}}$ and $H_{\mathrm{H}}$ scattering patterns have been qualitatively described in terms of a model of an isolated and symmetrical sector with its axis ("sheaf axis," $0 y_{3}$ ) oriented perpendicular to the ED (Figure 5(a). ${ }^{1}$ The sector is assumed to be optically anisotropic composed of uniaxially anisotropic scattering elements with polarizabilities $\alpha_{1}$ and $\alpha_{2}$ parallel and perpendicular to the principal optical axis $a$ (unit vector along the optical axis).

The outer four lobes (single hatched) of the $H_{\mathrm{V}}$ pattern with scattering maxima at $\theta=\theta_{\max , H_{\mathrm{V}}}$ and 
at azimuthal angles $\mu= \pm \mu_{\max }$ and $\pi \pm \mu_{\max }$ have been described in terms of the isolated sector model. The scattering angles $\theta_{\max , H_{\mathbf{V}}}$ and $\mu_{\max }$ of the maximum intensity are shown to be as-

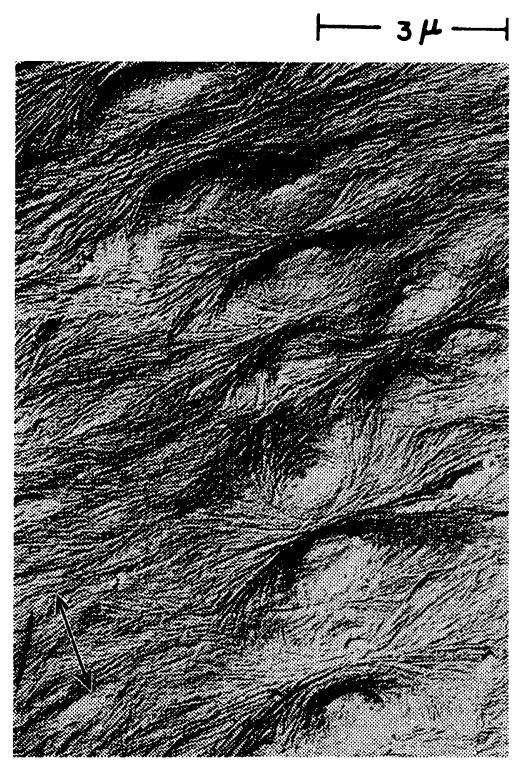

Figure 1. Typical electron micrograph of free specimen surfaces. Arrow indicates the extrusion direction. sociated with the average size of the sector $R_{0}$ and $\gamma_{0}{ }^{1}{ }^{1}$ The equatorial two lobes (single hatched) of the $V_{\mathrm{V}}$ and $H_{\mathrm{H}}$ patterns are also described in terms of an isolated model, the scattering angles $\theta_{\max , V_{\mathrm{V}}}^{\mu=90}$ and $\theta_{\max , H_{\mathrm{h}}}^{\mu=90}$ of the maximum intensity are reciprocally related to an "average distance" between the two opposing fans constituting the symmetrical sector.

The isolated model was shown to be unable to account for the inner four lobes (double hatched) of the $H_{\mathrm{V}}$ pattern with maximum intensity at $\mu= \pm\left[\pi / 2-\left(\gamma_{\mathrm{m}}\right)_{H_{\mathrm{V}}}\right]$ and $\pm\left[\pi / 2+\left(\gamma_{\mathrm{m}}\right)_{H_{\mathrm{V}}}\right]$ and those of the $V_{\mathrm{v}}$ pattern (no hatched). These are proposed to arise from an inter-sheaf interference of the scattered fields. The angles $\left(\gamma_{\mathrm{m}}\right)_{H_{\mathrm{V}}}$ and $\left(\gamma_{\mathrm{m}}\right)_{V_{\mathrm{v}}}$ are thought to be associated with the angle $\alpha_{0}$, i.e., an average orientation angle of rownuclei $^{1}$ with respect to ED. This prediction may be partly confirmed by the observation that the elongation of the specimen along the ED systematically decreases the angle $\left(\gamma_{\mathrm{m}}\right)_{H_{\mathrm{V}}}$ and $\left(\gamma_{\mathrm{m}}\right)_{V_{\mathrm{V}}}$, and thus the angle $\alpha_{0}$.

The isolated model also fails to account for the scattering maxima of the meridional two lobes (double hatched) of the $V_{\mathrm{V}}$ and $H_{\mathrm{H}}$ patterns. The scattering is considered to arise from the intersheaf interference effect, the scattering angles $\theta_{\max , V_{\mathbf{v}}}^{\mu=0}$

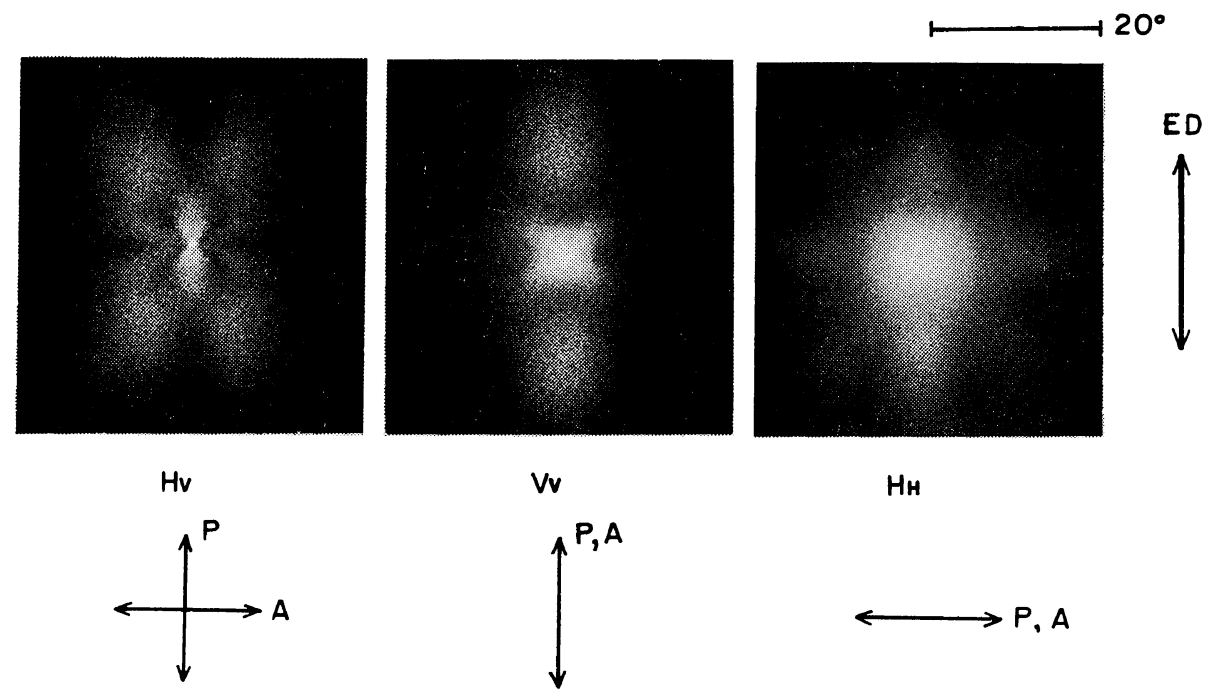

Fig. 2. Typical $H_{\mathrm{V}}, V_{\mathrm{V}}$, and $H_{\mathrm{H}}$ light-scattering patterns from the specimens. $\mathrm{P}$ and $\mathrm{A}$ indicate the polarization direction of the polarizer and analyzer, respectively. The extrusion direction (ED) is in vertical. 


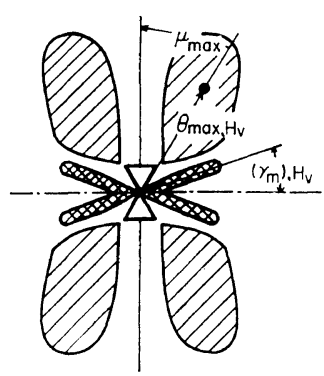

Hv

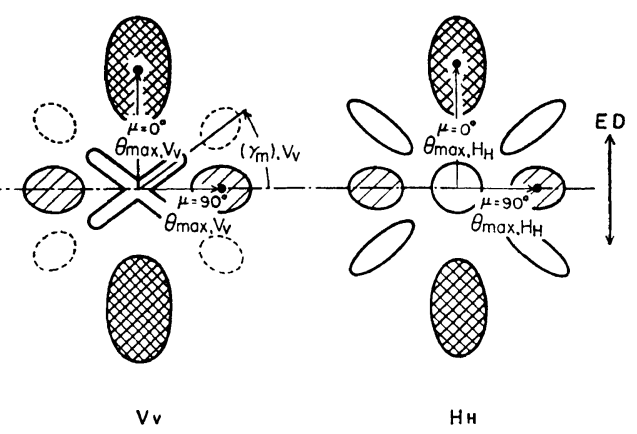

Figure 3. Schematic representations of the scattering patterns shown in Figure 2, and definitions of $\theta_{\max , H \mathrm{~V}}, \mu_{\max },\left(\gamma_{\mathrm{m}}\right)_{I I \mathrm{~V}}, \theta_{\max , V_{\mathrm{V}}}^{\mu=0}, \theta_{\mathrm{max}}^{\mu=90}, V_{\mathrm{V}},\left(\gamma_{\mathrm{m}}\right)_{V_{\mathrm{V}}}, \theta_{\mathrm{max}, H_{\mathrm{h}}}^{\mu=0}$, and $\theta_{\max , I I_{\mathrm{h}}}^{\mu=90}$.

and $\theta_{\max , H_{\mathrm{h}}}^{\mu=0}$ of the maximum intensity of which are reciprocally related to the average intersheaf distance, $\bar{d}^{1}$ The angle was shown to decrease systematically with elongation along the ED, indicating that the distance, $\bar{d}$ increases with elongation as may be expected.

In this article, we will generalize our earlier calculations on scattering from the isolated sheaf. We will consider scattering from an assembly of sheaves in which interference of the scattered fields from different sheaves should be taken into account.

\section{MODEL}

First to consider is the scattered intensity from the one-dimensional assembly of the anisotropic sheaves. The assembly contains $N$ particles (sheaves) and its orientation fluctuates around an average angle $\alpha_{0}$ with respect to the ED. For the sake of simplicity we shall assume that interference of the scattered fields from different assemblies can be ignored, i.e., only the interference of the scattered fields from the particles within an assembly being considered, and that the assemblies are oriented in a plane $0 y_{1} z_{1}$ perpendicular to propagation direction of the incident beam (Figure 4).

The sheaf is assumed to be a two-dimensional sector oriented in the plane of $0 y_{1} z_{1}$ as before. Orientation of the sheaf axis, $0 y_{3}$, fluctuates around its average orientation $\left(\xi=\xi_{0}\right.$, Figure $5(\mathrm{~b})$ ), though the fluctuations are assumed to be independent and not correlated. The displacement vector

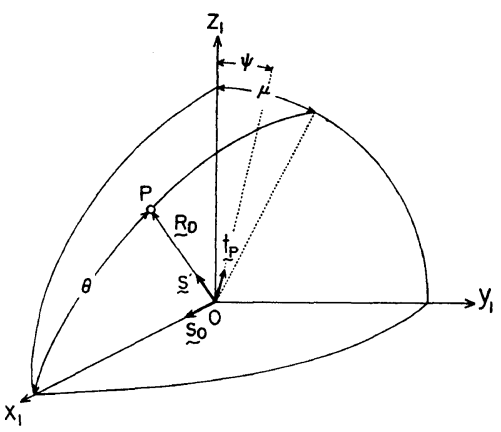

Figure 4. Definitions of the scattering angle $\theta$ and azimuthal angle $\mu$ and polarization direction $\psi$ of incident beam. The unit vectors $s_{0}$ and $s^{\prime}$ indicate the propagation directions of the incident and scattered beams, respectively, and $t_{\mathrm{p}}$ corresponds to that of the polarization direction of the incident beam. The $0 z_{1}$ axis coincides with the ED. The vector $\boldsymbol{R}_{\mathrm{D}}$ is a displacement vector between the scattering center and a point of observation.

between adjacent scattering centers are allowed to fluctuate around its average vector, $\bar{d}$ according to a particular distance statistics described by Hosemann. ${ }^{2}$ The fluctuation of the distance between adjacent particles is assumed to be independent, so that the probability, $H_{n}(z)$ of finding the $n$th neighbour at a distance, $z$ from a given particle is given by a convolution product of $H_{1}(z)$, the probability of finding the nearest neighbour at a distance $z$,

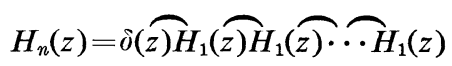

where $\delta$ is Dirac's delta function and $H_{1}$ occurs $(n-1)$ times. 


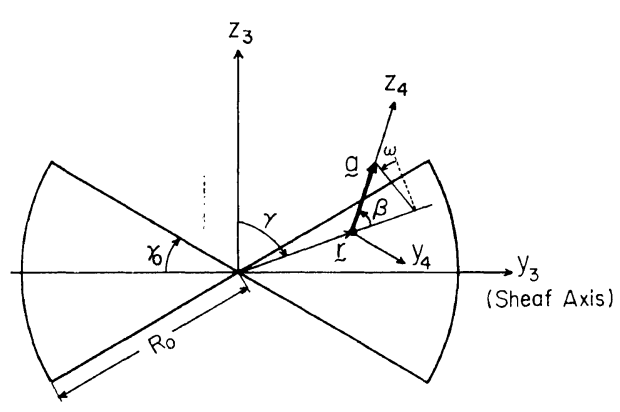

Figure 5. (a) Optically anisotropic sector as a model for the sheaflike crystalline superstructure. $R_{0}$ is the radius and $\gamma_{0}$ is half of the aperture angle of the sector. $\boldsymbol{a}$ is the unit vector along the principal optical axis of the uniaxially anisotropic scattering element. $x_{3} y_{3} z_{3}$ and $x_{4} y_{4} z_{4}$ are the coordinates fixed to the sheaf and the polarizability ellipsoid, respectively. The angles $\beta$ and $\omega$ specify orientation of the optical axis with respect to radius of the sheaf.

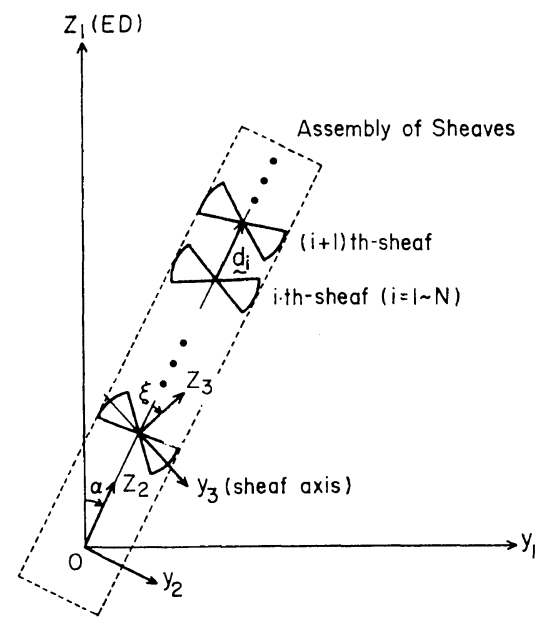

Figure 5. (b) Relationship among the coordinate systems $x_{1} y_{1} z_{1}$ (fixed to the apparatus), $x_{2} y_{2} z_{2}$ (fixed to the assembly) and $x_{3} y_{3} z_{3}$ (fixed to the sheaf). The angle $\alpha$ denotes orientation of the row-nucleus (or assembly axis, $\left.0 z_{2}\right)$ with respect to the $\operatorname{ED}\left(0 z_{1}\right)$ and $\xi$ denotes that of the sheaf with respect to the rownucleus $\left(0 z_{2}\right)$. The vector $\boldsymbol{d}_{i}$ is the displacement vector connecting the centers of the adjacent particles. The axes $0 x_{1}, 0 x_{2}$, and $0 x_{3}$ are coaxial.

Orientation of the row-nucleus or the axis of assembly, i.e., $0 z_{2}$ and that of the sheaf axis, i.e., $0 y_{3}$ are set to be symmetric with respect to the ED $\left(0 z_{1}\right.$ axis) and the transverse direction $\left(0 y_{1}\right.$ axis) so that the symmetry of the system matches to that of the observed morphology. The two dimensional model should suffice for our present purpose in studying qualitatively the interparticle interference effect in connection with possibly existing row nuclei.

\section{THEORY}

\section{Scattering from an Assembly}

First, consider the scattering intensity, $I(\boldsymbol{h}, \alpha)$ from a single assembly oriented at an angle $\alpha$ with respect to the ED. If $f_{j}(\boldsymbol{h}, \xi)$ is the scattering amplitude from the $j$-th sheaf in the assembly under a given polarization condition, and $\boldsymbol{z}_{j k}$ is a displacement vector between the $j$ - and $k$-th scattering centers, the scattered intensity at a point of observation located far from the scattering material is given by ${ }^{5}$

$$
I(\boldsymbol{h}, \alpha)=(c / 8 \pi) \sum_{j, k}\left\langle f_{j} f_{k}^{*} \exp \left(-i \boldsymbol{h} \cdot \boldsymbol{z}_{j k}\right)\right\rangle
$$

where $f^{*}$ denotes a complex conjugate of $f$, and $\boldsymbol{h}$ is the scattering vector defined by,

$$
\boldsymbol{h}=(2 \pi / \lambda)\left(s_{0}-s^{\prime}\right)=(2 \pi / \lambda) s
$$

$\lambda$ is the wavelength of light in the medium, and $c$ is the velocity of light in vacuo. The vectors $s_{0}$ and $s^{\prime}$ are unit vectors along the propagation directions of the incident and scattered beams, respectively. The factor $(c / 8 \pi)$ is associated with a coefficient related to the Poynting's theorem in the Gauss unit. ${ }^{5}$

The angular bracket $\langle>$ denotes an ensemble average, i.e., the average overall possible distributions of particles in the assembly with respect to spatial distributions of scattering centers and orientation of the individual particles. If there is no orientation correlation between the individual particles, and the orientation fluctuation is independent of the correlation in the position of the centers of each particle, it follows from eq 2 that

$$
\begin{aligned}
I(\boldsymbol{h}, \alpha)(8 \pi / c) \\
=N\left\langle|f|^{2}\right\rangle+\langle|f|\rangle^{2} \sum_{\substack{j, k \\
(j \neq k)}}\left\langle\exp \left(-i \boldsymbol{h} \cdot \boldsymbol{z}_{j k}\right)\right\rangle \\
=N\left[\left\langle|f|^{2}\right\rangle-|\langle f\rangle|^{2}\right] \\
+|\langle f\rangle|^{2}\left[N+\sum_{\substack{j, k \\
j \neq k}}\left\langle\exp \left(-\boldsymbol{i h} \cdot \boldsymbol{z}_{j k}\right)\right\rangle\right]
\end{aligned}
$$


The double summation may be rewritten as,

$$
\begin{gathered}
\sum_{\substack{j, k \\
(j \neq k)}}\left\langle\exp \left(-\boldsymbol{i h} \cdot \boldsymbol{z}_{j k}\right)\right\rangle=\sum_{j=1}^{N}\left[\sum _ { k = 1 } ^ { j - 1 } \left\langle\exp \left(-\boldsymbol{i} \boldsymbol{h} \cdot \boldsymbol{z}_{j k}\right)\right.\right. \\
\left.+\sum_{k=j+1}^{N}\left\langle\exp \left(-\boldsymbol{i} \boldsymbol{h} \cdot \boldsymbol{z}_{j k}\right)\right\rangle\right]
\end{gathered}
$$

where the first term in the right hand side of eq 5 is given by

$$
I_{1} \equiv \sum_{k=1}^{j-1}\left\langle\exp \left(-\boldsymbol{i h} \cdot \boldsymbol{z}_{j k}\right)\right\rangle=\sum_{k=1}^{j-1}\left\langle\prod_{i=k}^{j-1} \exp \left(-\boldsymbol{i} \boldsymbol{h} \cdot \boldsymbol{d}_{i}\right)\right\rangle
$$

where $\boldsymbol{d}_{i}$ is a displacement vector between $i$ and $(i+1)$ th particles. Since the distance fluctuation is independent, it follows that

$$
\begin{aligned}
I_{1} & =\sum_{k=1}^{j-1} \prod_{i=k}^{j-1}\left\langle\exp \left(-i \boldsymbol{h} \cdot \boldsymbol{d}_{i}\right)\right\rangle=\sum_{k=1}^{j-1} \prod_{i=k}^{j-1} F(\boldsymbol{h}) \\
& =\sum_{k=1}^{j-1} F^{j-k}=\left(F-F^{j}\right) /(1-F)
\end{aligned}
$$

where

$$
F(\boldsymbol{h}) \equiv\left\langle\exp \left(-\boldsymbol{i} \boldsymbol{h} \cdot \boldsymbol{d}_{i}\right)\right\rangle=\int H_{1}(\boldsymbol{z}) \exp (-\boldsymbol{i} \boldsymbol{h} \cdot \boldsymbol{z}) \mathrm{d} \boldsymbol{z}
$$

The function $H_{1}(z)$ is the probability of finding the nearest-neighbour particle at a displacement vector $\boldsymbol{z}$.

Similarly

$$
I_{2} \equiv \sum_{k=j+1}^{N}\left\langle\exp \left(i \boldsymbol{h} \cdot \boldsymbol{z}_{j k}\right)\right\rangle=\left[F^{*}-\left(F^{*}\right)^{N-j+1}\right] /\left[1-F^{*}\right]
$$

where $F^{*}$ is a complex conjugate of $F$, the Fourier transformation of the distance statistics, $H_{1}$. From eq $4-6$, and 8 , one obtains that

$$
(I / N)(8 \pi / c)=\left[\left\langle|f|^{2}\right\rangle-|\langle f\rangle|^{2}\right]+|\langle f\rangle|^{2}\left[Z_{1}+I_{c} / N\right]
$$

$$
\begin{array}{r}
Z_{1}=Z_{1}(\boldsymbol{h}, \alpha)=\operatorname{Re}[(1+F) /(1-F)] \\
I_{c}=I_{c}(\boldsymbol{h}, \alpha)=-2 \operatorname{Re}\left[F\left(1-F^{N}\right) /(1-F)^{2}\right]
\end{array}
$$

It is well known that the first term in the right-hand side of eq 9 is responsible for a diffuse background scattering of generally weak intensity arising from the orientation fluctuation of individual particles and distribution of particle size. But the second term, is associated with the paracrystalline order in the spatial arrangement of the scattering centers in the assembly $\left(\left|\langle f\rangle^{2}\right| Z_{1}\right)$ and with a zero-order scattering arising from the assembly as a whole, $\left(|\langle f\rangle|^{2} I_{c} / N\right)$.

The distance statistic $H_{1}(z)$ was first given by Prins $^{3,4}$ in the exponential form. In stead of using this function, we have adopted the function introduced by Hosemann,

$$
\begin{aligned}
H_{1}(z)= & (2 \pi)^{-3 / 2}\left(\Delta d_{z x}^{2} \Delta d_{z y}^{2} \Delta d_{z z}^{2}\right)^{-1 / 2} \\
& \times \exp \left\{-\frac{1}{2}\left[\frac{x_{2}{ }^{2}}{\Delta d_{z x}^{2}}+\frac{y_{2}{ }^{2}}{\Delta d_{z y}^{2}}+\frac{(z-\bar{d})^{2}}{\Delta d_{z z}^{2}}\right]\right\}
\end{aligned}
$$

where $\bar{d}$ is the average distance between adjacent particles, and $\Delta d_{z i}^{2}$ are the mean square fluctuations of the displacement vector $\boldsymbol{d}_{i}$ between the two adjacent particles in $i$-th direction where $i$ may be $x_{2}, y_{2}$, and $z_{2}$ axes (Figure 5(b)). The Fourier transformation of the function $H_{1}$ is given by ${ }^{2}$

$$
F(\boldsymbol{h})=|F(\boldsymbol{h})| \exp [-i(\boldsymbol{h} \cdot \overline{\boldsymbol{d}})]
$$

where

$$
F(\boldsymbol{h})=\exp \left[-\left(\Delta d_{z x}^{2} h_{x}{ }^{2}+J d_{z y}^{2} h_{y}{ }^{2}+\Delta d_{z z}^{2} h_{z}{ }^{2}\right) / 2\right]
$$

The quantities $h_{x}, h_{y}$, and $h_{z}$ are $x_{2}, y_{2}$, and $z_{2}$ components of the scattering vector $\boldsymbol{h}$.

In a special case where $\Delta d_{z x}^{2}=\Delta d_{z y}^{2}=0$, it follows that

$$
\begin{gathered}
H_{1}(z)=\left(2 \pi \Delta d_{z z}^{2}\right)^{-1 / 2} \exp \left[-\left(z_{2}-\bar{d}\right)^{2} / 2 \Delta d_{z z}^{2}\right] \\
|F(\boldsymbol{h})|=\exp \left(-\Delta d_{z z}^{2} h_{z}{ }^{2} / 2\right)
\end{gathered}
$$

In the limit of large distance fluctuation i.e., $\Delta d_{z z}^{2} \rightarrow \infty$, it follows that $|F| \rightarrow 0$, and therefore the lattice factor $Z_{1}$

$$
Z_{1}(\boldsymbol{h})=\left[1-|F|^{2}\right] /\left[1-2|F| \cos (\boldsymbol{h} \cdot \overline{\boldsymbol{d}})+|F|^{2}\right]
$$

approaches unity and the zero-order scattering $I_{c}(\boldsymbol{h})$

$$
\begin{aligned}
I_{c}(\boldsymbol{h})= & -2|F|\left\{\left[1+|F|^{2}\right] \cos (\boldsymbol{h} \cdot \overline{\boldsymbol{d}})-2|F|\right. \\
& -|F|^{N} \cos [(N+1) \boldsymbol{h} \cdot \overline{\boldsymbol{d}}] \\
& +2|F|^{N+1} \cos [N(\boldsymbol{h} \cdot \overline{\boldsymbol{d}})] \\
& \left.-|F|^{N+2} \cos [(N-1) \boldsymbol{h} \cdot \overline{\boldsymbol{d}}]\right\} / \\
& {\left[1-2|F| \cos (\boldsymbol{h} \cdot \overline{\boldsymbol{d}})+|F|^{2}\right]^{2} }
\end{aligned}
$$

approaches zero. Consequently the scattered intensity in the limiting case is given by eq 9 , 17 , and 18 as, 


$$
I / N=(c / 8 \pi)\left\langle|f|^{2}\right\rangle, \text { when } \Delta d_{z z}^{2} \rightarrow \infty
$$

Therefore the scattering in the limiting case becomes a criterion of so-called "independent" scattering.

\section{Scattering from Individual Particles}

The scattered field $\boldsymbol{\Delta} \boldsymbol{E}_{\mathrm{s}}$ from a volume element $\mathrm{d} r$ within a given sector is expressed, at the point of observation $\boldsymbol{R}_{\mathrm{D}}$ far from the center of scattering material, by ${ }^{5}$

$$
\Delta \boldsymbol{E}_{\mathrm{s}}(\boldsymbol{r}, t)=E_{0}\left(k_{0}{ }^{2} / R_{\mathrm{D}}\right)\left[\boldsymbol{s}^{\prime} \times \boldsymbol{s}^{\prime} \times \boldsymbol{M}\right] \exp [i(\boldsymbol{h} \cdot \boldsymbol{r})] \mathrm{d} \boldsymbol{r}
$$

where $E_{0}$ is the magnitude of the electric field vector $\boldsymbol{E}_{0} \exp (i \omega t)$ of the incident beam at the volume element $\mathrm{d} r$, and $k_{0}=2 \pi / \lambda_{0}, \lambda_{0}$ being the wavelength of light in vacuo. The vector $\boldsymbol{M}=$ $\boldsymbol{M}(\boldsymbol{r}, t)$ is the oscillating dipole moment induced by unit strength of the incident field $E_{0}$ and is given in a form,

$$
\boldsymbol{M}(\boldsymbol{r}, t)=\boldsymbol{M}_{1}(\boldsymbol{r}) \exp \left[i \omega\left(t-R_{\mathrm{D}} / c\right)\right]
$$

where $c$ is the velocity of light in vacuo. The amplitude $\boldsymbol{M}_{1}(\boldsymbol{r})$ having a spatial fluctuation in scattering material depends upon the size, shape, and relative orientation of the polarizability ellipsoid of the volume element $\mathrm{d} \boldsymbol{r}$ with respect to the incident field vector $\boldsymbol{E}_{0}$, as will be discussed in the following. It was assumed that the variation of the optical properties of the volume element with time occurs slowly compared with the time-scale of the optical frequencies, so that variation in the amplitude $\boldsymbol{M}_{1}$ with time may be ignored. The last term exp $[i(\boldsymbol{h} \cdot \boldsymbol{r})]$ is the phase factor of the scattered field from the element $d r$ relative to the scattering center.

Depolarized and polarized compoments of scattered light are detected by observing the scattered light through an analyzer. If the polarization direction of the analyzer is specified by a unit vector $\boldsymbol{O}$ the total scattered field $f(\boldsymbol{h})$ from a sheaf is given by

$$
\begin{aligned}
f(\boldsymbol{h}) & =\int \mathrm{d} r\left[\Delta E_{\mathrm{s}}(\boldsymbol{r}) \cdot \boldsymbol{O}\right] \\
& =\int_{\pi / 2-r_{0}}^{\pi / 2+r_{0}} \mathrm{~d} \gamma \int_{0}^{R_{0}} r \mathrm{~d} r\left[\Delta \boldsymbol{E}_{\mathrm{s}}(\boldsymbol{r}) \cdot \boldsymbol{O}\right]
\end{aligned}
$$

The scattering amplitude $f$ thus obtained cor- responds to the function $f$ in eq 9. We assume that scattering from a large particle can be described in terms of the Rayleigh-Gans-Born or Rayleigh-Debye theories. ${ }^{5}$ The assumption is, of course, legitimate in our problem.

The induced dipole moemnt $\boldsymbol{M}(\boldsymbol{r})$ at the point $\boldsymbol{r}$ is given in terms of the coordinate system fixed to the polarizability ellipsoid of the scattering element, i.e., $0 x_{4} y_{4} z_{4}$ coordinate (Figure 5(a)), as follows:

$$
M_{1}=\alpha Q E_{0} / E_{0}=\alpha Q t_{\mathrm{p}}
$$

$t_{\mathrm{p}}$ is a unit vector along the polarization direction of incident beam, and $\boldsymbol{\alpha}$ is the polarizability tensor of the scattering element, relative to the medium surrounding the sheaf, i.e.,

$\boldsymbol{\alpha}=\left(\begin{array}{ccc}b_{\mathrm{t}} & 0 & 0 \\ 0 & b_{\mathrm{t}} & 0 \\ 0 & 0 & b_{\mathrm{r}}\end{array}\right), b_{\mathrm{t}}=\alpha_{2}-\alpha_{\mathrm{s}}, b_{\mathrm{r}}=\alpha_{1}-\alpha_{\mathrm{s}}$

It is assumed that the scattering element has uniaxial anisotropy with polarizabilities $\alpha_{1}$ and $\alpha_{2}$ along and perpendicular to the principal optical axis and that the surrounding medium is isotropic, having polarizability $\alpha_{\mathrm{s}} . \boldsymbol{Q}$ is an orthogonal matrix of the coordinate transformation from $0 x_{1} y_{1} z_{1}$ to $0 x_{4} y_{4} z_{4}$, and $\boldsymbol{E}_{0}$ is the amplitude of the electric field of the incident beam whose direction is given in the $0 x_{1} y_{1} z_{1}$ coordinate system by a column vector

$$
\boldsymbol{t}_{\mathrm{p}}=(0, \sin \psi, \cos \psi)
$$

where the angle $\psi$ characterizes the polarization direction of the incident beam (Figure 4).

The induced dipole moment $\boldsymbol{M}_{1}$ described by $x_{4}, y_{4}$, and $z_{4}$ components can be represented in terms of $x_{1}, y_{1}$, and $z_{1}$ components by multiplying $\boldsymbol{M}_{1}$ in eq 23 by the transposed matrix of $\boldsymbol{Q}$.

$$
M_{1}={ }^{t} Q \alpha Q t_{\mathrm{p}}
$$

The matrix of the coordinate transformation $Q$ may be given by

$$
\boldsymbol{Q}=\boldsymbol{Q}_{3} \boldsymbol{Q}_{2} \boldsymbol{Q}_{1},{ }^{\mathrm{t}} \boldsymbol{Q}={ }^{\mathrm{t}} \boldsymbol{Q}_{1}{ }^{\mathrm{t}} \boldsymbol{Q}_{2}{ }^{\mathrm{t}} \boldsymbol{Q}_{3}
$$

where $\boldsymbol{Q}_{i}$ is the transformation matrix of the coordinate from $x_{i} y_{i} z_{i}$ to $x_{i+1} y_{i+1} z_{i+1}(i=1-3)$. From Figure 5, these matrices are obviously given by 
Light Scattering by Films Crystallized from Oriented Melts

$$
\begin{aligned}
& \boldsymbol{Q}_{1}=\left(\begin{array}{ccc}
1 & 0 & 0 \\
0 & \cos \alpha & -\sin \alpha \\
0 & \sin \alpha & \cos \alpha
\end{array}\right) \\
& \boldsymbol{Q}_{2}=\left(\begin{array}{ccc}
1 & 0 & 0 \\
0 & \cos \xi & -\sin \xi \\
0 & \sin \xi & \cos \xi
\end{array}\right) \\
& \boldsymbol{Q}_{3}=\left(\begin{array}{ccc}
t^{-1} & -t u v & -t u w \\
0 & t w & -t v \\
u & v & w
\end{array}\right)
\end{aligned}
$$

where

$$
\begin{aligned}
u & =\sin \beta \sin \omega \\
v & =\cos \beta \sin \gamma-\sin \beta \cos \omega \cos \gamma \\
w & =\cos \beta \cos \gamma+\sin \beta \cos \omega \sin \gamma \\
t & =\left(v^{2}+w^{2}\right)^{-1 / 2}
\end{aligned}
$$

In determining $Q_{3}, 0 z_{4}$ is taken parallel to the optical axis, $a$, and $0 y_{4}$ is perpendicular to $a$ and in the plane of $0 y_{3} z_{3}$. The angles $\beta$ and $\omega$ are the polar and azimuthal angles of the otpical axis $a$ with respect to radius of the sheaf $\boldsymbol{r}$.

From eq $20-22$, and 26-31, one obtains that

$$
\begin{aligned}
f(\boldsymbol{h})= & \left(\frac{2 \pi}{\lambda_{0}}\right)^{2} \frac{E_{0}}{R_{\mathrm{D}}} \exp \left[i \omega\left(t-\frac{R_{\mathbf{D}}}{c}\right)\right] \\
& \times \int_{\pi / 2-\gamma_{0}}^{\pi / 2+r_{0}} \mathrm{~d} r\left(\boldsymbol{O} \cdot\left[\boldsymbol{s}^{\prime} \times\left(\boldsymbol{s}^{\prime} \times \boldsymbol{M}_{1}\right)\right]\right) \\
& \times \int_{0}^{R_{0}} r \mathrm{~d} r \exp (i \boldsymbol{h} \cdot \boldsymbol{r})
\end{aligned}
$$

where

$$
\boldsymbol{h} \cdot \boldsymbol{r}=-k r \sin \theta \cos (\xi+\alpha-\mu+\gamma)
$$

It should be noted that $\boldsymbol{M}_{1}=\boldsymbol{M}_{1}(\boldsymbol{r})=\boldsymbol{M}_{1}(r, \gamma)$ is independent of $r$ but dependent only upon the angle $\gamma$ for a homogeneous and anisotropic sector for which $\alpha_{1}, \alpha_{2}, \beta$ and $\omega$ characterizing optical properties of the sector are constant through the space. Furthermore, in case of $\omega=0$ (planar orientation) or a randomly varying $\omega$, the particle is centersymmetric. Thus, the phase factor $\exp [i(\boldsymbol{h} \cdot \boldsymbol{r})]$ becomes simply $\cos (\boldsymbol{h} \cdot \boldsymbol{r})$. In such a case, it follows that

$$
\begin{aligned}
\int_{0}^{R_{0}} r \mathrm{~d} r \cos (h \cdot r)= & R_{0}{ }^{2}\left[\frac{\sin W_{\mathrm{s}} R_{0}}{W_{\mathrm{s}} R_{0}}\right. \\
& \left.-\frac{1}{2} \frac{\sin ^{2}\left(W_{\mathrm{s}} R_{0} / 2\right)}{\left(W_{\mathrm{s}} R_{0} / 2\right)^{2}}\right]
\end{aligned}
$$

where

$$
W_{\mathrm{s}} R_{0}=W \cos (\xi+\alpha+\gamma-\mu), W=\left(2 \pi R_{0} / \lambda\right) \sin \theta
$$

The rest of the integral in eq 32 must be numerically calculated. As a special case of interest, $\omega=0$, which we are going to deal with in this article, the integrand is given by

$$
\begin{aligned}
\left(\mathbf { 0 } \cdot \left[\boldsymbol{s}^{\prime}\right.\right. & \left.\left.\times\left(\boldsymbol{s}^{\prime} \times \boldsymbol{M}_{1}\right)\right]\right)_{\omega=0} \\
= & \delta E_{0}\left\{\operatorname { s i n } \psi \left[\left(\mathbf{0} \cdot \hat{\boldsymbol{y}}_{1}\right) \sin ^{2}(\hat{\xi}+\alpha+\gamma-\beta)+\left(\mathbf{0} \cdot \hat{\boldsymbol{z}}_{1}\right)\right.\right. \\
& \times \sin 2(\xi+\alpha+\gamma-\beta)] \\
& +\cos \psi[(\mathbf{0} \cdot \hat{\boldsymbol{y}}) \sin 2(\xi+\alpha+\gamma-\beta) \\
& \left.+\left(\mathbf{0} \cdot \hat{\boldsymbol{z}}_{1}\right) \cos ^{2}(\xi+\alpha+\gamma-\beta)\right] \\
& \left.+p\left[\left(\mathbf{0} \cdot \hat{\boldsymbol{y}}_{1}\right) \sin \psi+\left(\mathbf{0} \cdot \hat{\boldsymbol{z}}_{1}\right) \cos \psi\right]\right\}
\end{aligned}
$$

wher $p$ is defined by

$$
p=\left(\alpha_{2}-\alpha_{\mathrm{s}}\right) /\left(\alpha_{1}-\alpha_{2}\right)=b_{\mathrm{t}} / \delta
$$

Polarized and Depolarized Components of the Scattered Light

The unit vector along the polarization direction of the analyzer 0 was defined by Cabannes ${ }^{6}$ in such a way that (i) for the prarallel polarizer and analyzer, i.e., $\mathbf{0}=\mathbf{0}_{/ /}, \mathbf{0}_{/ /}$should be in the plane of $\boldsymbol{s}^{\prime}$ and $\boldsymbol{t}_{\mathrm{p}}$, and be perpendicular to $\boldsymbol{s}^{\prime}$ and that (ii) for the crossed polarizer and analyzer, $\mathbf{0}=\mathbf{0}$, $\mathbf{0}_{+}$should be perpendicular to the plane of $\boldsymbol{s}^{\prime}$ and $\boldsymbol{t}_{\mathrm{p}}$. These are given as follow, ${ }^{7,8}$

$$
\begin{aligned}
\mathbf{0}_{/ /}= & -\left[\cos ^{2} \theta+\sin ^{2} \theta \sin ^{2}(\psi-\mu)\right]^{-1 / 2} \\
& \times\left\{\sin \theta \cos \theta \cos (\psi-\mu) \hat{\boldsymbol{x}}_{1}\right. \\
& +\left[\sin ^{2} \theta \sin \mu \cos (\psi-\mu)-\sin \psi\right] \hat{\boldsymbol{y}}_{1} \\
& \left.+\left[\sin ^{2} \theta \cos \mu \cos (\psi-\mu)-\cos \psi\right] \hat{\boldsymbol{k}}_{1}\right\} \\
\mathbf{0}_{+}= & {\left[\cos ^{2} \theta+\sin ^{2} \theta \sin ^{2}(\mu-\psi)\right]^{-1 / 2} } \\
& \times\left\{\sin \theta \sin (\mu-\psi) \hat{\boldsymbol{x}}_{1}\right. \\
& \left.+\cos \theta \cos \psi \hat{\boldsymbol{y}}_{1}-\cos \theta \sin \psi \hat{\boldsymbol{z}}_{1}\right\}
\end{aligned}
$$

Apparently, the $V_{\mathrm{V}}, H_{\mathrm{V}}, V_{\mathrm{H}}, H_{\mathrm{H}}$ scattering are obtained by setting the $\mathbf{0}$-vector as $\mathbf{0}_{/ /}\left(\psi=0^{\circ}\right)$, $\mathbf{0}_{+}\left(\psi=0^{\circ}\right), \mathbf{0}_{+}\left(\psi=90^{\circ}\right)$, and $\mathbf{0}_{/ /}\left(\psi=90^{\circ}\right)$, respectively.

\section{Orientation Distribution}

The scattered intensity $I=I(h, \alpha)$ in eq 9 and the particle scattering amplitude $f(h, \alpha, \xi)$ are calculated for a given orientation $\alpha$ of the assembly or nucleus, $0 z_{2}$ axis, and for a given orientation 
$\xi$ of the sheaf, $0 z_{3}$. We considered two extreme cases in terms of the orientation distributions for the nucleus $p_{1}(\alpha)$ and that for the sheaf axis $p_{2}(\xi)$.

(i) There are no orientation fluctuations of the sheaf axes within the assembly, though the assembly as a whole has some orientation fluctuations with respect to $0 z_{1}$, i.e.,

$$
p_{1}(\alpha)=\exp \left[-\sigma_{\alpha}^{2}\left(\sin \alpha-\sin \alpha_{0}\right)^{2}\right], \alpha_{0}=35^{\circ}
$$

and

$$
p_{2}(\xi)=\delta\left(\xi-\xi_{0}\right), \xi_{0}=-35^{\circ}
$$

where $\delta$ is Dirac's delta function.

(ii) There are no orientation fluctuations of the assembly axes as a whole, but the sheaf axes within the assembly have some independent orientation fluctuations, i.e.,

$$
p_{1}(\alpha)=\delta\left(\alpha-\alpha_{0}\right), \alpha_{0}=35^{\circ}
$$

and

$$
p_{2}(\xi)=\exp \left[-\sigma_{\xi}^{2}\left(\sin \xi-\sin \xi_{0}\right)^{2}\right], \xi_{0}=-35^{\circ}
$$

In the former case, the scattering intensity $I(\boldsymbol{h}, \alpha)$ from an assembly oriented at an angle $\alpha$ is given from eq 9 by

$$
\begin{aligned}
I(\boldsymbol{h}, \alpha) / N= & (\boldsymbol{c} / 8 \pi)\left|f\left(\boldsymbol{h}, \alpha, \hat{\xi}_{0}\right)\right|^{2} \\
& \times\left[Z_{1}(\boldsymbol{h}, \alpha)+I_{\mathrm{c}}(\boldsymbol{h}, \alpha) / N\right]
\end{aligned}
$$

The average intensity from the system is finally given by

$$
I(\boldsymbol{h})=\int_{0}^{2 \pi} \mathrm{d} \alpha I(\boldsymbol{h}, \alpha) p_{1}(\alpha) / \int_{0}^{2 \pi} \mathrm{d} \alpha p_{1}(\alpha)
$$

In case when $N \rightarrow \infty$ and $Z_{1} \rightarrow 1$ as in the case of large disorders,

$$
\begin{aligned}
I(h) & \rightarrow(c / 8 \pi) N\left\langle|f|^{2}\right\rangle \\
& =N\left(\frac{c}{8 \pi}\right) \int_{0}^{2 \pi} \mathrm{d} \alpha|f|^{2} p_{1}(\alpha) / \int_{0}^{2 \pi} \mathrm{d} \alpha p_{1}(\alpha)
\end{aligned}
$$

The independent scattering intensity thus obtained is given by a more explicit formula, by substituting eq 32 and 34 into eq 46 ,

$$
I(\boldsymbol{h}) / N \rightarrow \frac{I_{0}}{{R_{\mathrm{D}}}^{2}}\left(\frac{2 \pi}{\lambda_{0}}\right)^{4} F\left(w_{\mathrm{s}}, \mu\right)
$$

where $I_{0}$ is the intensity of the incident beam given by $(c / 8 \pi) E_{0}^{2}$, and

$$
\begin{aligned}
F\left(w_{\mathrm{s}}, \mu\right)= & R_{0}{ }^{4} \int_{0}^{2 \pi} \mathrm{d} \alpha p_{1}(\alpha) \\
& \times\left\{\int_{\pi / 2-\gamma_{0}}^{\pi / 2+\gamma_{0}} \mathrm{~d} \gamma\left(\mathbf{0} \cdot\left[s^{\prime} \times\left(s^{\prime} \times M_{1}\right)\right]\right)\right. \\
& \left.\times\left[\frac{\sin W_{\mathrm{s}} R_{0}}{W_{\mathrm{s}} R_{0}}-\frac{1}{2} \frac{\sin ^{2}\left(W_{\mathrm{s}} R_{0} / 2\right)}{\left(W_{\mathrm{s}} R_{0} / 2\right)^{2}}\right]\right\}^{2} / \\
& \int_{0}^{2 \pi} \mathrm{d} \alpha p_{1}(\alpha)
\end{aligned}
$$

where $\xi=\xi_{0}$.

In the latter case,

$$
\begin{aligned}
I(\boldsymbol{h}) / N= & (\boldsymbol{c} / 8 \pi)\left\{\left\langle\left|f\left(\boldsymbol{h}, \alpha_{0}, \xi\right)\right|^{2}\right\rangle-\left|\left\langle f\left(\boldsymbol{h}, \alpha_{0}, \xi\right)\right\rangle\right|^{2}\right. \\
& \left.+\left|\left\langle f\left(\boldsymbol{h}, \alpha_{0}, \xi\right)\right\rangle\right|^{2}\left[Z_{1}\left(\boldsymbol{h}, \alpha_{0}\right)+\boldsymbol{I}_{c}\left(\boldsymbol{h}, \alpha_{0}\right) / N\right]\right\}
\end{aligned}
$$

where

$$
\begin{aligned}
\left\langle\left|f\left(\boldsymbol{h}, \alpha_{0}, \xi\right)\right|^{2}\right\rangle= & \int_{0}^{2 \pi} \mathrm{d} \xi p_{2}(\xi)\left|f\left(\boldsymbol{h}, \alpha_{0}, \xi\right)\right|^{2} \mid \\
& \int_{0}^{2 \pi} \mathrm{d} \xi p_{2}(\xi) \\
\left|\left\langle f\left(\boldsymbol{h}, \alpha_{0}, \xi\right)\right\rangle\right|^{2}= & \mid \int_{0}^{2 \pi} \mathrm{d} \xi p_{2}(\xi) f\left(\boldsymbol{h}, \alpha_{0}, \xi\right) / \\
& \left.\int_{0}^{2 \pi} \mathrm{d} \xi p_{2}(\xi)\right|^{2}
\end{aligned}
$$

\section{Distribution of the Assembly Size}

In order to study the effect of size of the assembly $N \bar{d}$ ( $\bar{d}$ being the average intersheaf distance), on the scattering intensity distribution, $I_{c} / N$ in the second term of right-hand side of eq 9 was included. Size distribution of the assembly or distribution of $N$ in the assembly is also considered by assuming the distribution function $P(N)$ previously used in one of our papers, ${ }^{9}$

$$
P(N)=\exp \left[-\frac{(N-\bar{N})^{2}}{2 \sigma_{N}{ }^{2}}\right] / \sum_{N=1}^{2 \bar{N}-1} \exp \left[-\frac{(N-\bar{N})^{2}}{2 \sigma_{N}{ }^{2}}\right]
$$

where $\bar{N}$ and $\sigma_{N}$ are average number of the particles in the assembly and its standard deviation. The average zero-order scattering $\left\langle I_{\mathrm{c}}\right\rangle_{\mathrm{av}}$ is then given by

$$
\left\langle I_{\mathrm{c}}\right\rangle_{\mathrm{av}}=\sum_{N=1}^{2 \bar{N}-1} I_{\mathrm{c}}(N) P(N)
$$




\section{NUMERICAL CALCULATIONS AND RESULTS}

Numerical calculations of light-scattering intensity distribution from the system were performed for a special case of $\omega=0^{\circ}$ and $\beta=90^{\circ}$ (tangential orientation of the optical axis $a$ in the plane of the sector). All calculations were performed for the sectors with $R_{0}=3 \mu$ and $\gamma_{0}=30^{\circ}$ from the results obtained in a earlier work. ${ }^{1}$

The disorder in the interparticle distance is assumed to occur only along the assembly axis, $0 z_{2}$, so that

$$
\Delta d_{z x}^{2}=\Delta d_{z y}^{2}=0, \Delta d_{z z}^{2}=\Delta d^{2}
$$

The $z_{2}$ component of $\boldsymbol{h}$ in eq 16 is given by

$$
h_{\mathrm{z}}=\boldsymbol{h} \cdot \hat{\boldsymbol{z}}_{2}=-(2 \pi / \lambda) \sin \theta \cos (\alpha-\mu)
$$

by utilizing eq 28 .

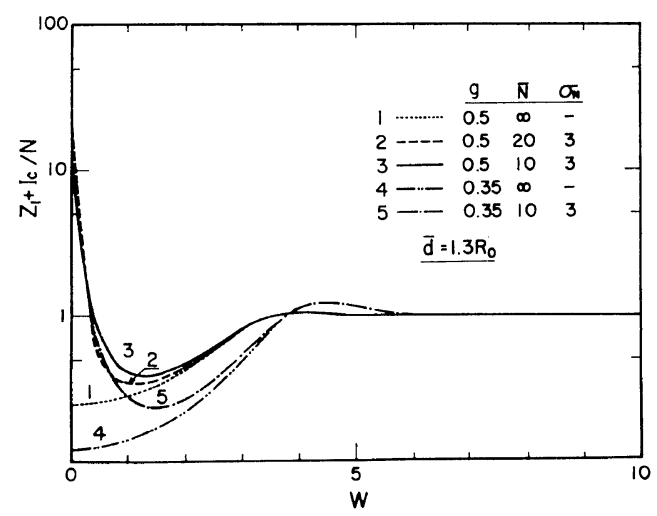

Figure 6. Angular dependences of calculated $\left(Z_{1}+I_{\mathrm{c}}\right)$ $N)$ along the assembly axis $(\mu=\alpha)$ for an assembly oriented at angle $\alpha$ with respect to $W=2 \pi\left(R_{0} / \lambda\right) \sin \theta$. $\bar{d}=1.3 R_{0}$.

Figure 6 shows angular dependences of calculated $\left(Z_{1}+I_{\mathrm{c}} / N\right)$ along the assembly axis, i.e., at $\mu=\alpha$ for a given assembly oriented at an angle $\alpha$ with respect to $0 z_{1}$ axis. The lattice and zeroorder scattering factors $\left(Z_{1}+I_{\mathrm{c}} / N\right)$ are plotted as a function of a reduced scattering angle $W=2 \pi\left(R_{0}\right)$ i) $\sin \theta$ for various sets of the parameters. The angular distribution was calculated for a particular value of average interparticle distance $\bar{d}=1.3 R_{0}$, a lattice disordered parameter $g=\Delta d / \bar{d}=0.5$ or 0.35 , an average number of particles in the assembly $\bar{N}=10,20$, and $\infty$, and its standard deviation $\sigma_{N}=3$.
Figure 7 shows (a) $H_{\mathrm{V}}, V_{\mathrm{V}}$, and $H_{\mathrm{H}}$ light scattering patterns for an isolated anisotropic sheaf with its axis $\left(0 y_{3}\right)$ perfectly oriented perpendicular to the vertical direction of the apparatus $\left(0 z_{1}\right.$ axis) (i.e., $\alpha=\xi=0$ ), and (b) the corresponding patterns for an assembly perfectly oriented along the vertical direction (i.e., $\alpha=0$ ) with $\bar{N}=10$, $\sigma_{N}=3, \quad \bar{d}=1.3 R_{0}, \quad g=0.5$, and $\xi=\xi_{0}=0^{\circ} \quad$ (i.e., within the assembly, the sheaf axes are perfectly oreinted perpendicular to the assembly axis). The value of $p$ in eq 37 was assumed to be $-1 / 3$. This corresponds to the case in which scattering arises predominantly from the orientation and anisotropy fluctuations rather than from density fluctuations associated with the term $\left(\alpha_{2}-\alpha_{\mathrm{s}}\right)$ and thus with the third term in eq 36.

Figure $8(\mathrm{a})$ represents the $H_{\mathrm{V}}, V_{\mathrm{V}}$, and $H_{\mathrm{H}}$ patterns for the assemblies of infinitely large size, i.e., $N=\infty$, and Figure 8 (b) represents those for assemblies of finite size, $\bar{N}=10$. In both cases, the assemblies are oriented at particular angles $\alpha_{0}= \pm 35^{\circ}$ with respect to the vertical or extrusion direction. No orientational disorders are introduced in terms of orientation of the assembly axis and of the sheaf axis. Again, it is assumed that $\bar{d}=1.3 R_{0}, g=0.5, p=-1 / 3$ and $\sigma_{N}=3$.

Figure 9(a) shows the effect of orientation distribution of the assembly and sheaf axes on the $H_{\mathrm{V}}, V_{\mathrm{V}}$, and $H_{\mathrm{H}}$ scattering patterns for the case (ii) in which the sheaf axes have some orientation fluctuations with in the assemblies oriented at particular angles $\alpha_{0}= \pm 35^{\circ}$ with respect to the ED, while Figure 9(b) shows that for case (i) in which the sheaf axes have a fixed orientation, $\xi_{0}=-35^{\circ}$, though the assembly as a whole has some orientation fluctuations. It was again assumed that $\bar{d}=1.3 R_{0}, g=0.5, p=-1 / 3, \bar{N}=10$, $\sigma_{N}=3$ and $\sigma=5$. The assumed orientation distribution functions $p_{1}(\alpha)$ and $p_{2}(\xi)$ are shown in Figure 10. We assumed that $\xi_{0}=-35^{\circ}$ from the electron microscopic evidence and $\alpha_{0}=35^{\circ}$ to attain the best fit between the calculated and observed zero-order scattering patterns as discussed later. The values for $\sigma=\sigma_{\alpha}=\sigma_{\xi_{0}}=5$ are arbitrarily assumed.

\section{DISCUSSIONS}

As shown in Figure 6, the zero-order scattering associated with scattering from the assembly as a 

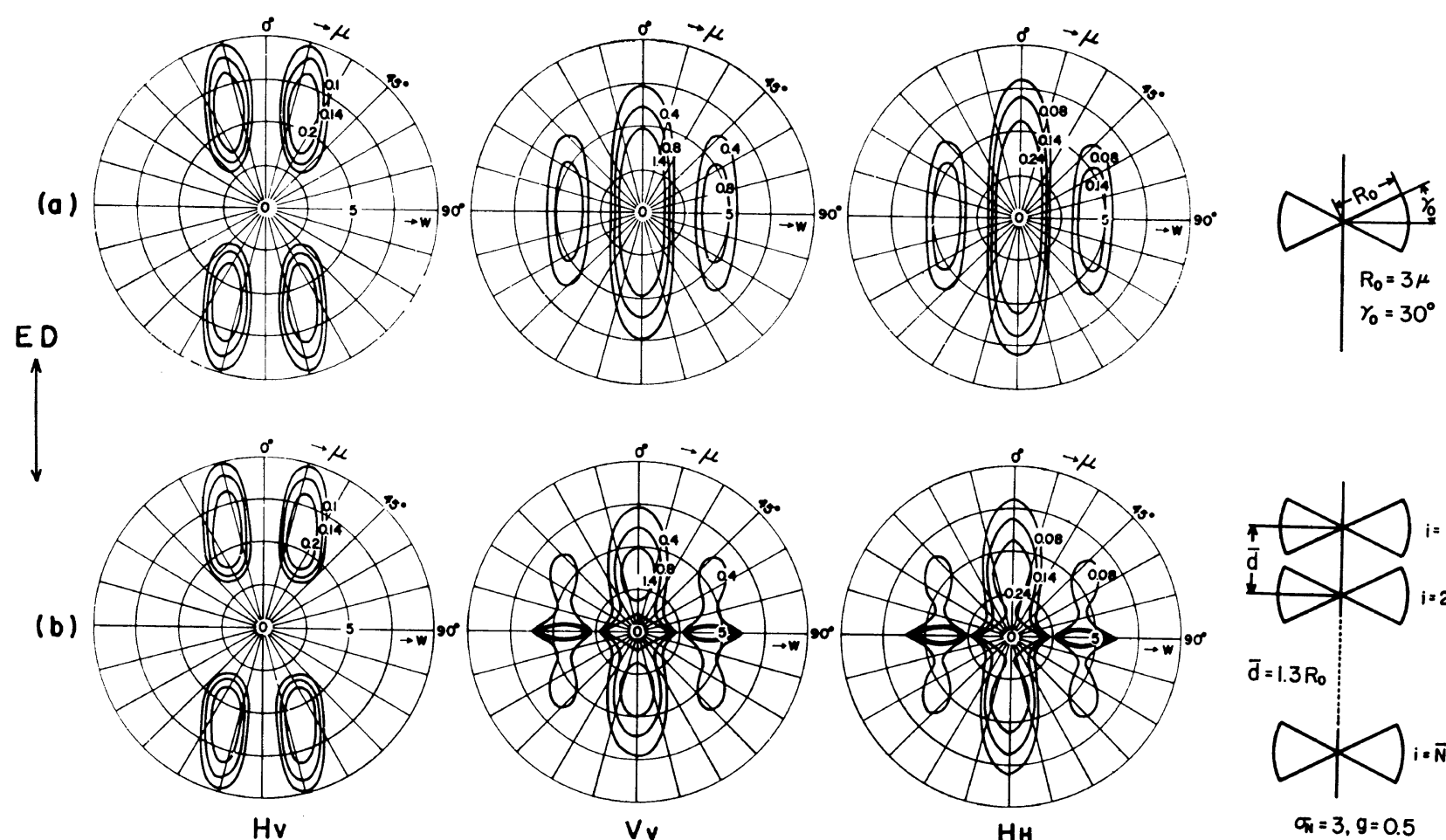

Figure 7. Calculated $H_{\mathrm{V}}, V_{\mathrm{V}}$, and $H_{\mathrm{H}}$ light-scattering patterns for (a) an isolated anisotropic sheaf with its axis $0 y_{3}$ oriented perpendicular to the $\mathrm{ED}$ and for (b) an assembly perfectly oriented along the vertical direction $(\alpha=0)$ with $\bar{N}=10, \sigma_{\mathrm{N}}=3$, $g=0.5$, and $\xi=0^{\circ}, p=-1 / 3$. The $\mathrm{ED}$ is in vertical. 


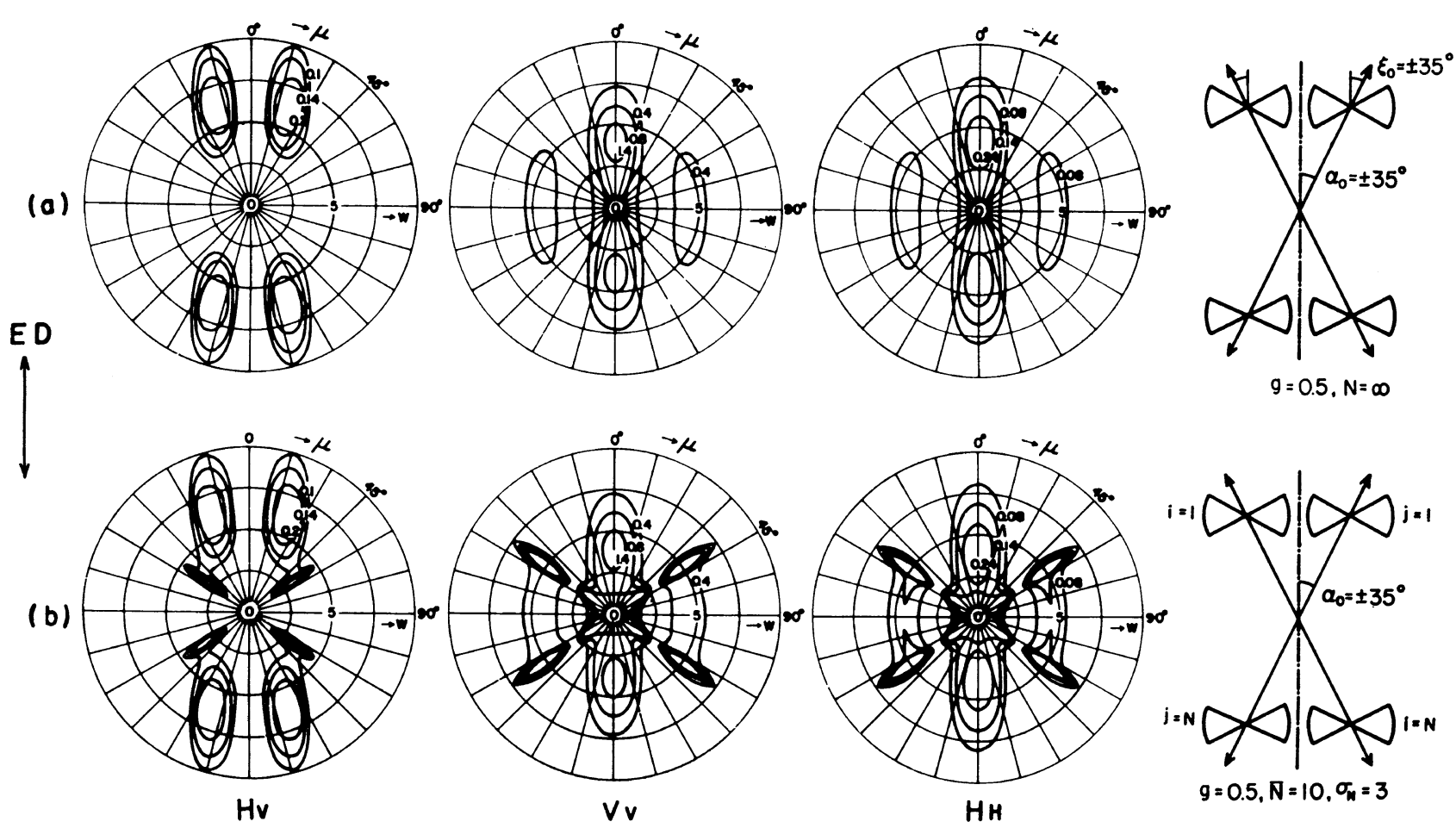

Figure 8. Calculated $H_{\mathrm{V}}, V_{\mathrm{V}}$, and $H_{\mathrm{H}}$ patterns for the assemblies of (a) $\bar{N}=\infty$ and of (b) $\bar{N}=10, \sigma_{\mathrm{N}}=3$. In both cases the assembly axes are oriented at particular angles $\alpha_{0}= \pm 35^{\circ}$ with respect to the ED which is in vertical direction. $\bar{d}=1.3 R_{0}$, $g=0.5, p=-1 / 3$. 


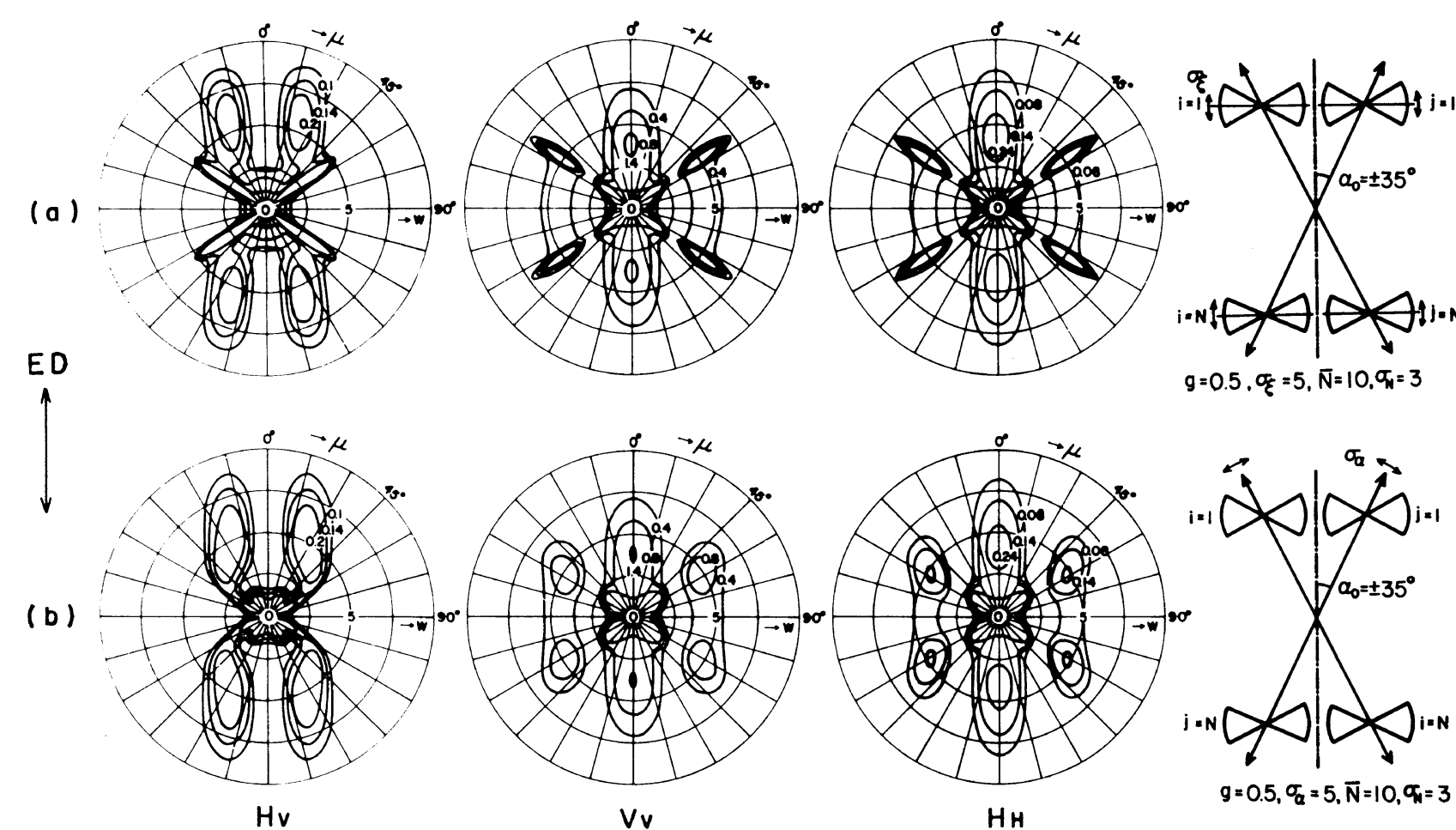

Figure 9. Calculated $H_{\mathrm{V}}, V_{\mathrm{V}}$, and $H_{\mathrm{H}}$ patterns from (a) the system having orientation fluctuations of the sheaf axis within the assembly and from (b) that having orientation fluctuations of the assembly axes. $\bar{d}=1.3 R_{0}, g=0.5, p=-1 / 3, \bar{N}=10, \sigma_{x}=$ 3 and $\sigma=5$. 


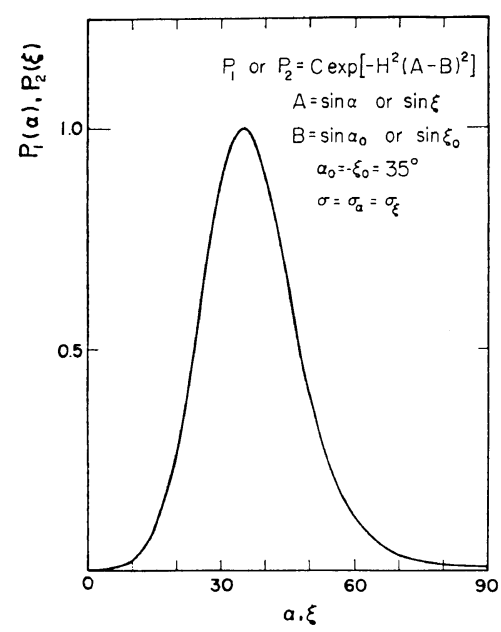

Figure 10. Assumed orientation distribution functions for the assembly axes, $p_{1}(\alpha)$ and the sheaf axes, $p_{2}(\xi) . \quad \alpha_{0}=-\xi_{0}=35^{\circ}, \sigma=\sigma_{\alpha}=\sigma_{\xi_{0}}=5$.

whole, i.e., the term associated with $I_{\mathrm{c}} / N$ is important in general only at very small scattering angles, typically at $W$ smaller than 2 for the size of the assembly studied in this article. This may be clearly seen by comparison of curves 1 to 3 and curves 4 and 5 . At larger scattering angles, the lattice factor $Z_{1}$ associated with regularity in the spatial distribution of the interparticle distance plays an important role. As pointed out by Hosemann, ${ }^{2}$ only the first order maximum is discernible at the scattering angle reciprocally related to the average interparticle distance $\bar{d}$ (i.e., $W_{\mathrm{m}}=$ $2 \pi\left(R_{0} / \lambda\right) \times \sin \theta \simeq 2 \pi R_{0} / \bar{d}=2 \pi / 1.3=4.8, \quad$ noticing that $2 \bar{d} \sin (\theta / 2)=\lambda)$ for systems having the disorder parameter $g, 0.18 \lesssim g \lesssim 0.35$. The greater interparticle disorders, as in the case of $g=0.5$, smears out the maximum in $Z_{1}$. The lattice factor $Z_{1}$ approaches unity at scattering angles greater than $W_{\mathrm{m}} \simeq 4.8$. At these angles the scattering from the system becomes identical to independent scattering as already discussed. It may be noted in the figure that

$$
\lim _{\theta \rightarrow 0} Z_{1}=g^{2}, \lim _{\theta \rightarrow 0} I_{\mathrm{c}} / N=N-g^{2}
$$

so that $\left(Z_{1}+I_{\mathrm{c}} / N\right) \rightarrow N$ as $\theta \rightarrow 0$, which is theoretically confirmed from eq 10 and 11 .

Figure 7(a) shows scattering patterns from an isolated particle perfectly oriented with its axis $0 y_{3}$ normal to the ED or $0 z_{1}$ direction. The $H_{\mathrm{v}}$,
$V_{\mathrm{V}}$, and $H_{\mathrm{H}}$ patterns from the isolated particle qualitatively agree with the experimental patterns shown in Figures 2 and 3. The four lobes of the $H_{\mathrm{V}}$ pattern have their maximum intensity at $W=W_{\max }$ and $\mu=\mu_{\max }$. These angles are related to the size and shape of the sector as described in a earlier paper. ${ }^{1}$ The corresponding patterns in Figure 7(b) are those from 10 sheaves, aligned parallel to the ED with $\bar{d}=1.3 R_{0}$, and $g=0.5$. The $H_{\mathrm{V}}$ pattern is not affected much by the interference, while the $V_{\mathrm{V}}$ and $H_{\mathrm{H}}$ patterns are significantly affected in that (i) the interparticle interference gives rise to the scattering maximum at the meridian in the $V_{\mathrm{V}}$ and $H_{\mathrm{H}}$ scattering, as was found in the experimental patterns, and that (ii) the interference also gives a strong zero-order scattering for the $V_{\mathrm{V}}$ and $H_{\mathrm{H}}$ patterns on the equa tor owing to scattering from the assembly as a whole. Thus, it is obvious that a better agree ment between the calculated and experimental patterns may be obtained by introducing the effect of interparticle interference.

The value of $\bar{d}=1.3 R_{0} \approx 4 \mu$ is assigned, since it gives maximum intensity in the meridional $V_{\mathrm{v}}$ and $H_{\mathrm{H}}$ scattering intensity distributions at the scattering angles, as observed in the experimental patterns, i.e., at the $\theta_{\max , V_{\mathrm{v}}}^{\mu=0}$ and $\theta_{\max , H_{\mathrm{h}}}^{\mu=0}$ (see Figure 3), respectively. The $g$-value and the values for $\bar{N}$ and $\sigma_{N}$ are not uniquely determined. A precise determination of the parameters requires a more quantitative comparison between the calculated and measured intensity distributions, and is beyond the scope of this study. There are no higher order maxima in the intensity distributions of the meridional $V_{\mathrm{V}}$ and $H_{\mathrm{H}}$ scattering, indicating that the $g$-value should be greater than 0.35 , probably around 0.5 . The value of $\bar{N}$ of order 10 seems to fit of the calculated and observed patterns.

The $H_{\mathrm{V}}$ patterns are not affected much by interference because at the scattering angles where the particle-scattering factor $|f|^{2}$ is not zero, the lattice factor $Z_{1}$ becomes almost unity. The strong zero-order scattering from the assembly as a whole cannot be expected in the $H_{\mathrm{V}}$ patterns, contrary to the cases of the $V_{\mathrm{V}}$ and $H_{\mathrm{H}}$ patterns since the particle factor $|f|^{2}$ becomes zero under the $H_{\mathrm{V}}$ polarization at the small scattering angles.

The calculated patterns still deviate from the 
experimentals patterns in that (i) the calculated $H_{\mathrm{V}}$ pattern does not give the zero-order scattering, while the corresponding experimental pattern exhibits the inner four lobes (double hatched) at $\mu= \pm\left[\pi / 2 \pm\left(\gamma_{\mathrm{m}}\right)_{H_{\mathrm{v}}}\right]$ and meridional two lobes (see Figure 3 ), and that (ii) the calculated zero-order scattering in $V_{\mathrm{V}}$ and $H_{\mathrm{H}}$ patterns are oriented on the equator, while those found in the experimental patterns are oriented nearly at a diagonal direction as seen in Figures 2 and 3.

To account for the discrepancies between the caclulated and experimental patterns, one may consider the case where the nuclei or the assembly axes are oriented at particular angles $\pm \alpha_{0}$ with respect to the ED. Figures 8 and 9 represent the scattering patterns corresponding to this case. For such a complex system, it may be worthwhile to describe separately the contribution of each factor, i.e., the particle $|f|^{2}$, the lattice $Z_{1}$ and the zero-order scattering factor $\left(I_{\mathrm{c}} / N\right)$ to the net scattering. Figure 11 represents the contour plots of (a) $Z_{1}$, (b) $I_{\mathrm{c}} / N$, and (c) $Z_{1}+I_{\mathrm{c}} / N$ for the case in which the assembly orients perfectly either at angle $\alpha_{0}$ or at angle $-\alpha_{0}, \alpha_{0}=35^{\circ}$ and for $\bar{d}=1.3 R_{0}$ and $g=0.5$. As indicated above, there is no interference effect between the scattering fields from different assemblies.

At a given azimuthal angle, $Z_{1}$ is minimum at $W=0$ and generally increases with increasing $W$, passing a broad and ill-defined maximum (region with double hatch) and then decreases to an asymptotic value of 2 except at $\mu= \pm 55^{\circ}$ for which the asymptotic value is $1+g^{2}=1.25$. This is because the value $Z_{1}$, for each assembly, increases to unity with an increase in the scattering angle $W$ and the system is composed of the two assemblies oriented at $\alpha= \pm \alpha_{0}$. For $\mu= \pm 55^{\circ}$, however, only one assembly contributes to the angular dependence of $Z_{1}$ and the other orients normal to the scanning plane, i.e., $\alpha-\mu= \pm 90^{\circ}$ in eq 55 for the other assembly, thus leading to value $Z_{1}=g^{2}=0.25$ independent of scattering angles. The function $Z_{1}$ at a given $W$ passes through a minimum value at azimuthal angle $\mu= \pm 55^{\circ}$, i.e., at the angles reciprocally related to orientation of the assembly. The scattering from the system becomes a criterion of the independent scattering at the single hatched region where the net value of $Z_{1}$ approaches the asymptotic values of 2 or 1.25 . The double hatched region corresponds to the angular region where $Z_{1}$ passes through a very broad and ill-defined maximum.

The zero-order scattering associated with scattering from the assembly as a whole has a steep peak intensity at $W=0$ and continuously decreases with increasing $W$ at a given azimuthal angle shown in Figure 11(b) for $\bar{N}=10$ and $\sigma_{N}=3$. The scattering intensity $I_{\mathrm{c}} / N$ passes through steep peaks at $\mu= \pm 55^{\circ}$, i.e., at the angle reciprocally

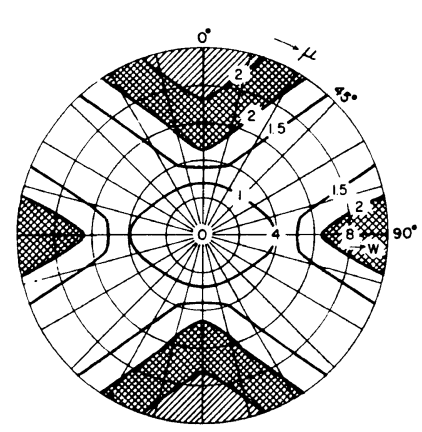

$\mathrm{Z}_{1}$

(a)

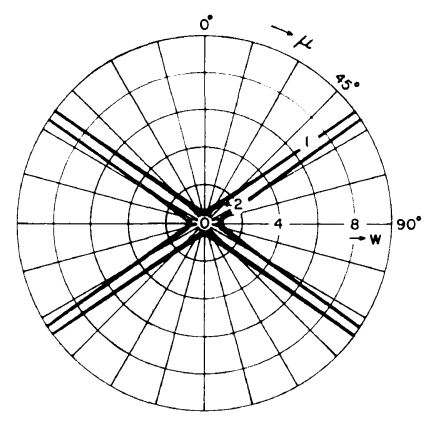

$\mathrm{I}_{\mathrm{C}} / \mathrm{N}$

(b)

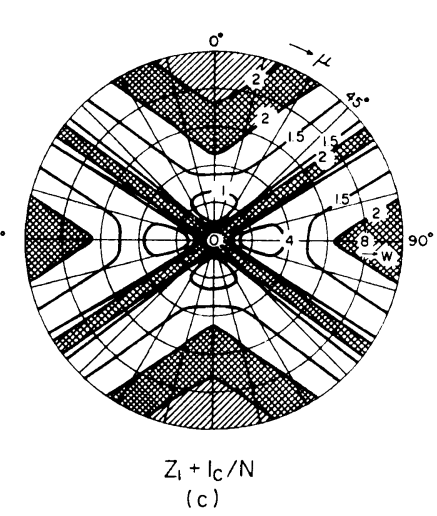

(c)

Single Hatch $-Z_{1}$ : Indep. Scattering

Double Hatch - Max. $Z_{1} \& I_{c} / N$

Figure 11. Contour plots of (a) $Z_{1}$, (b) $I_{\mathrm{c}} / N$, and (c) $Z_{1}+I_{\mathrm{c}} / N$ for the case where the assemblies orient at angles $\pm 35^{\circ}$ with the ED. $Z_{1}$ and $Z_{1}+I_{\mathrm{c}} / N$ are equal to 2 at the single hatched region where the scattering falls into a criterion of the independent scattering. Intensity becomes maximum at the double hatched region. 
related to the orientation of the assemblies.

The scattering patterns shown in Figure 8(a) are therefore interpreted as a product of the corresponding patterns shown in Figure 7(a) and the contour pattern in Figure 11(a), while those shown in Figure 8(b) are interpreted as a product of the patterns in Figure 7(a) and the pattern in Figure 11(c). The meridional scattering maxima in the $V_{\mathrm{V}}$ and $H_{\mathrm{H}}$ patterns are primarily determined by the meridonal intensity distribution of the lattice factor $Z_{1}$. The diagonal zero-order scattering lobes shown in Figure 8(b) with maximum intensity at $\mu= \pm\left[\pi / 2 \pm\left(\gamma_{\mathrm{m}}\right)_{H_{\mathrm{V}}}\right]$ for the $H_{\mathrm{V}}$ scattering and at $\mu= \pm\left[\pi / 2 \pm\left(\gamma_{\mathrm{m}}\right)_{V_{\mathrm{V}}}\right]$ for the $V_{\mathrm{V}}$ scattering (see Figure 3) are attributed to the strong angular dependence of $I_{\mathrm{c}} / N$ as shown in Figure 11(b). The best fit of the observed and calculated patterns in the zero-order scattering region was obtained for the value of $\alpha_{0}= \pm 35^{\circ}$.

The results obtained so far indicate that the inclination of the row nuclei with the ED best explains the experimental scattering patterns. However, close observation of the results may indicate that the calculated patterns still deviate from the observed patterns in that (i) no scattering exists at the center of the calculated $H_{\mathrm{V}}$ patterns,

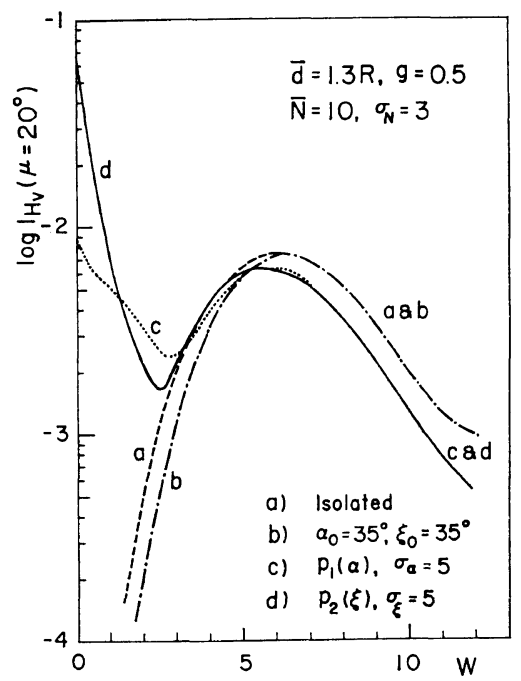

Figure 12. $H_{\mathrm{V}}$ intensity distribution at $\mu=20^{\circ}$ as a function of $W=(2 \pi / \lambda) R_{0} \sin \theta$ for various scattering models; the curves a to $\mathrm{d}$ correspond to scattering from the models shown in Figures $7(\mathrm{a}), 8(\mathrm{~b})$, 9(b), and 9(a), respectively. $\bar{d}=1.3 R_{0}, g=0.5, \bar{N}=$ 10 , and $\sigma_{N}=3$. and that (ii) the four lobes oriented in diagonal directions in the calculated $H_{\mathrm{v}}$ and $V_{\mathrm{v}}$ patterns have angular dependence(s) with $\mu$ which are too strong in comparison with those of the experimental patterns. In order to improve the defects in the model one may consider an effect of orientation distribution of the individual particle, $p_{2}(\xi)$, and of the assembly axis or nucleus, $p_{1}(\alpha)$, around a particular direction as discussed earlier.

The effects of the orientation distribution of the particles and the assembly axes or nuclei are shown in Figures 9(a) and 9(b), respectively. On introducing orientation fluctuations, the calculated patterns become even closer to the observed patterns. The calculated $H_{\mathrm{V}}$ pattern is improved in that the fluctuation yields finite intensity at the center of the pattern. This even explains the inner two lobes in meridian as found experimentally (see $H_{\mathrm{V}}$ pattern in Figure 9(b)). The finite intensity at the center is attributed to non-vanishing scattered intensity $|\langle f\rangle|^{2}$ from the individual particle as is expected when the orientation fluctuation is introduced. The fluctuation also improves the $V_{\mathrm{V}}$ and $H_{\mathrm{H}}$ patterns in that it decreases the strong angular dependence of the four lobes oriented in the diagonal directions of the patterns. The model giving rise to the scattering patterns shown in Figure 9(b) seems to be most adequate among those studied in this article. It should be noted
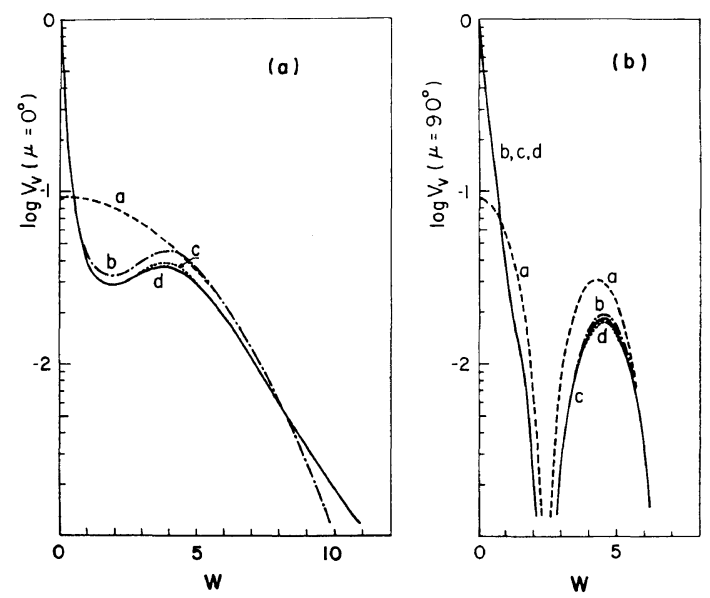

Figure 13. $V_{\mathrm{v}}$ intensity distributions at $\mu=0^{\circ}$ (a) and $\mu=90^{\circ}$ (b) as a function of $W$ for the various models. The curves a to $d$ have the same meanings as those in Figure 12. 
that the intensity of the $V_{\mathrm{V}}$ and $H_{\mathrm{H}}$ patterns at $W \approx 5$ and $\mu \approx 60$ is only slightly higher than that at $W \approx 5$ and $\mu \approx 90^{\circ}$. A greater orientational disorder may account for the equatorial scattering lobes (single hatched) as depicted in Figure 3.

The interparticle interference effect may be seen in a more quantitative manner by examining the intensity distribution itself. Figures 12 and 13 represent, respectively, the effect on the angular dependence of the $H_{\mathrm{V}}$ pattern with $W$ at a particular azimuthal angle $\mu$ and that of the $V_{\mathrm{V}}$ pattern at $\mu=0^{\circ}$ and $90^{\circ}$ for the same parameters as in Figures 7 to 9 , i.e., $\bar{d}=1.3 R_{0}, g=0.5, \bar{N}=10$ and $\sigma_{N}=3$. The curve, a, corresponds to the scattering from an isolated particle. The curve, $b$, represents the scattering from the assemblies in which there are no orientation fluctuations of the nuclei and sheaf axes, while the curves $c$ and $d$ correspond, respectively, to the cases where orientation of either the sheaf axis or the assembly axis fluctuates.

It is clearly shown in Figures 12 and 13 that, at large scattering angles, the interparticle interference tends to average out, thus leading to the result that the scattered intensity from the assembly divided by the number of particles within it becomes identical to the scattered intensity from the isolated particle. The curves $a$ and $b$ differ from the $c$ and $d$ curves at the large scattering angles, and this is attributed to the difference in the particle scattering factor caused by different orientation distribution rather than by interference phenomenon (Figures 12 and 13(a)).

The angular dependences of curves $b$ to $d$ differ very much from the curve, $a$, at small scattering angles owing to the interparticle interference effect. The zero-order scattering gives a large excess scattering intensity at very small scattering angles, $W$ less than 1 . The intensity minimum in the meridional $V_{\mathrm{V}}$ intensity distribution at $W \simeq 2$ primarily arises from the contribution associated with the lattice factor $Z_{1}$.

\section{CONCLUSIONS}

The butterfly-type $H_{\mathrm{V}}$ pattern was found to be typical of the scattering from oriented sheaf-like crystalline superstructure (or probably from flattened spherulites, ${ }^{10}$ though this possibility can be ruled out in our case) resulting from lamellar overgrowth from rows of nucleating points and the subsequent lamellar branchings. Measurements of $\theta_{\max , H_{\mathrm{V}}}$ and $\mu_{\max }$ of the butterfly type pattern, and $\theta_{\mathrm{max}, V_{\mathrm{V}}}^{\mu=90}$ and $\theta_{\mathrm{max}, H_{\mathrm{h}}}^{\mu=90}$ of the polarized components of the scattering enable an estimation of the shape and size of the sheaf and its changes during crystallization and deformation processes.

The maxima in the meridional $V_{\mathrm{V}}$ and $H_{\mathrm{H}}$ scattering intensity distributions arise essentially from an interparticle interference effect. The position of the scattering maxima $\theta_{\max , V_{\mathrm{V}}}^{\mu=0}$ and $\theta_{\max , H_{\mathrm{h}}}^{\mu=0}$ are reciprocally related to the average intersheaf distance. The inner four lobes of the $H_{\mathrm{V}}$ (double hatched) and $V_{\mathrm{V}}$ pattern are attributed to the zero-order scattering from the assemblies as a whole, oriented preferentially at inclined angles $\alpha_{0}= \pm 35^{\circ}$ with respect to the ED. The angles are closely related to the orientation of the inner four lobes, $\left(\gamma_{\mathrm{m}}\right)_{H_{\mathrm{V}}}$ and $\left(\gamma_{\mathrm{m}}\right)_{V_{\mathrm{V}}}$, and may be related to orientation of the row nuclei with respect to the ED. The biaxial orientation of the row nuclei or the assembly axes may reflect a biaxial flow of melt and therefore a biaxial molecular orientation involved in a particular tubular extrusion processing. The meridional two lobes (non hatched) in the $H_{\mathrm{V}}$ pattern occur as a consequence of the orientation distribution of the sheaf axes and/or the nuclei, internal disorders of the type as described by Stein and coworkers $^{11-13}$ and asymmetry in the shape of the sheaflike crystalline superstructure.

\section{REFERENCES}

1. T. Hashimoto, A. Todo, Y. Murakami, and H. Kawai, J. Polym. Sci., Polym. Phys. Ed., 15, 501 (1977).

2. R. Hosemann and S. N. Bagchi, "Direct Analysis of Diffraction by Matter", North-Holland Publishing Co., Amsterdam, 1962.

3. F. Zernicke and J. A. Prins, Z. Phys., 41, 184 (1927).

4. J. Frenkel, "Kinetic Theory of Liquids," Dover, New York, N.Y., 1955.

5. See for examples, H.C. van de Hulst, "Light Scattering by Small Particles," John Wiley \& Sons, Inc., New York, N.Y., 1957; M. Kerker, "The Scattering of Light and Other Electromagnetic Radiation," Academic Press, New York, N.Y., 1969; B. Chu, "Laser Light Scattering," Academic Press., New York, N.Y., 1974; J. A. Stratton, 
"Electromagnetic Theory," Mcgraw-Hill, New York, N.Y., 1941.

6. J. Cabannes, "La Diffusion Moleculaire de la Lumiere," Les Presses Universitaries de France, Paris, 1929.

7. A. E. M. Keijzers, J. J. van Aartsen, and W. Prins, J. Am. Chem. Sco., 90, 3107 (1968).

8. J. J. van Aartsen and R. S. Stein, J. Polym. Sci., Part A-2, 9, 295 (1971).

9. T. Hashimoto, K. Nagatoshi, A. Todo, H. Hasegawa, and H. Kawai, Macromolecules, 7, 364
(1974).

10) V. G. Baranov, S. Ya. Frenkel, V. I. Gromov, T. I. Volkov, and R. S. Zurabian, J. Polym. Sci., Part C, 38, 61 (1972).

11. R. S. Stein and W. Chu, J. Polym. Sci., Part $A-2,8,1137$ (1970).

12. T. Hashimoto and R. S. Stein, J. Polym. Sci., Part A-2, 9, 1747 (1971).

13. D. Y. Yoon and R. S. Stein, J. Polym. Sci., Polym. Phys. Ed., 12, 763 (1974). 